\title{
Gcd-closed sets and determinants of matrices associated with arithmetical functions
}

\author{
by
}

\section{Shaofang Hong (Chengdu)}

1. Introduction. Smith [11] proved that if $f$ is an arithmetical function and $[f(i, j)]$ is the $n \times n$ matrix having $f$ evaluated at the greatest common divisor of $i$ and $j$ as its $i, j$-entry, then

$$
\operatorname{det}[f(i, j)]=(f * \mu)(1)(f * \mu)(2) \ldots(f * \mu)(n),
$$

where $\mu$ is the Möbius function and $f * \mu$ is the Dirichlet convolution of $f$ and $\mu$. Apostol [2] extended Smith's result by showing that if $f$ and $g$ are arithmetical functions and if $\beta$ is defined for positive integers $t$ and $r$ by $\beta(t, r)=\sum_{d \mid(t, r)} f(d) g(r / d)$, then $\operatorname{det}[\beta(i, j)]=[g(1)]^{n} f(1) \ldots f(n)$. He noted that as a consequence, $\operatorname{det}[C(i, j)]=n$ !, where $C(t, r)$ is Ramanujan's sum. Paul McCarthy [8] generalized Smith's and Apostol's results to the class of even functions $(\bmod r)$. He evaluated the determinants of $n \times n$ matrices of the form $[\beta(i, j)]$, where $\beta(t, r)$ is an even function of $t(\bmod r)$. A complex-valued function $\beta(t, r)$ of the positive integral variables $t$ and $r$ is said to be an even function of $t(\bmod r)$ if $\beta(t, r)=\beta((t, r), r)$ for all values of $t$. The functions considered by Smith and Apostol are even functions of $t(\bmod r)$ for every $r$. Bourque and Ligh [3] evaluated the determinants of $n \times n$ matrices of the form $\left[\beta\left(x_{i}, x_{j}\right)\right]$, where the set $S=\left\{x_{1}, \ldots, x_{n}\right\}$ of distinct positive integers is factor-closed (i.e., $S$ contains every divisor of $x$ for any $x \in S)$ and $\beta(t, r)$ is an even function of $t(\bmod r)$.

Throughout this paper, let $S=\left\{x_{1}, \ldots, x_{n}\right\}$ be a set of distinct positive integers. The set $S$ is said to be gcd-closed if $\left(x_{i}, x_{j}\right) \in S$ for $1 \leq i, j \leq n$. Clearly, a factor-closed set is gcd-closed but not conversely. Let $f(t), g(t)$ and $h(t)$ be arithmetical functions. The $\Psi(t, r)$ is defined for all positive

2000 Mathematics Subject Classification: 11C20, 11A25.

Key words and phrases: gcd-closed set, arithmetical function, Ramanujan's trigonometric sum, determinant.

Supported partially by the NNSF of China (Grant No. 10101015), the Postdoctoral Science Foundation of China and the YSRF of Sichuan University. 
integers $t$ and $r$ as follows:

$$
\Psi(t, r)=\sum_{d \mid(t, r)} f(d) g\left(\frac{t}{d}\right) h\left(\frac{r}{d}\right) .
$$

Define the class of arithmetical functions $L_{S}=\left\{l(t): l\left(d_{1} / d\right)=l\left(d_{2} / d\right)\right.$ whenever $d \mid d_{1}$ for any $d_{1}, d_{2} \in S$ satisfying $\left.d_{1} \mid d_{2}\right\}$. In 1993, Bourque and Ligh [3] evaluated the determinant of the $n \times n$ matrix $\left[\Psi\left(x_{i}, x_{j}\right)\right]$ if the set $S=\left\{x_{1}, \ldots, x_{n}\right\}$ is factor-closed. In this paper we will evaluate the determinant of the $n \times n$ matrix $\left[\Psi\left(x_{i}, x_{j}\right)\right]$, where $S=\left\{x_{1}, \ldots, x_{n}\right\}$ is gcd-closed and $g \in L_{S}$ or $h \in L_{S}$. As applications, we evaluate the determinants of $n \times n$ matrices of the form $\left[C\left(x_{i}, x_{j}\right)\right]$, where $S=\left\{x_{1}, \ldots, x_{n}\right\}$ is gcd-closed, and $C(t, r)$ is Ramanujan's trigonometric sum. These results generalize Bourque and Ligh's results [3]. We also evaluate the determinant of $n \times n$ matrix $\left[\frac{g}{f * g}\left(\frac{x_{i}}{\left(x_{i}, x_{j}\right)}\right)\right]$, where $f$ is completely multiplicative, $g(m)=\mu(m) h(m), h$ is multiplicative, $f(p) \neq 0$ and $f(p) \neq h(p)$ for all primes $p$, and $(f * g)(d) \neq 0$ for any positive integer $d$ satisfying $d \mid x, x \in S$, and $S=\left\{x_{1}, \ldots, x_{n}\right\}$ is gcd-closed.

2. Determinant of the matrix $\left[\Psi\left(x_{i}, x_{j}\right)\right]$. In the present section, we evaluate the determinant of the $n \times n$ matrix $\Psi\left(x_{i}, x_{j}\right)$, where $g \in L_{S}$ or $h \in L_{S}$ and $S=\left\{x_{1}, \ldots, x_{n}\right\}$ is gcd-closed.

Lemma 1 ([3]). Let $T=\left\{y_{1}, \ldots, y_{m}\right\}$ be a factor-closed set containing $S$. Then $\left[\Psi\left(x_{i}, x_{j}\right)\right]=G \Lambda H^{\mathrm{T}}$, where $\Lambda=\operatorname{diag}\left(f\left(y_{1}\right), \ldots, f\left(y_{m}\right)\right)$ and the $n \times m$ matrices $G$ and $H$ are defined by $G=\left[g\left(x_{i} / y_{j}\right)\right]$ and $H=\left[h\left(x_{i} / y_{j}\right)\right]$, respectively.

Lemma 2. Let the set $S=\left\{x_{1}, \ldots, x_{n}\right\}$ of distinct positive integers be gcd-closed. If $g \in L_{S}$ or $h \in L_{S}$ then there exist $n \times n$ lower triangular matrices $M$ and $N$ with diagonal elements 1 and an $n \times n$ lower triangular matrix $P$ with diagonal elements

$$
\sum_{d \mid x_{1}} f(d) g\left(\frac{x_{1}}{d}\right) h\left(\frac{x_{1}}{d}\right), \sum_{\substack{d \mid x_{2} \\ d \nmid x_{1}}} f(d) g\left(\frac{x_{2}}{d}\right) h\left(\frac{x_{2}}{d}\right), \ldots, \sum_{\substack{d \mid x_{n} \\ d \nmid x_{l}, x_{l}<x_{n}}} f(d) g\left(\frac{x_{n}}{d}\right) h\left(\frac{x_{n}}{d}\right),
$$

such that $\left[\Psi\left(x_{i}, x_{j}\right)\right]=M P N^{\mathrm{T}}$.

Proof. Without loss of generality we may let $1 \leq x_{1}<\ldots<x_{n}$. Let $S_{k}=\left\{d: d \in \mathbb{Z}^{+}, d \mid x_{k}, d \nmid x_{t}, t<k\right\}, 1 \leq k \leq n$. Clearly $S_{k_{1}} \cap S_{k_{2}}=\emptyset$ for $1 \leq k_{1}, k_{2} \leq n, k_{1} \neq k_{2}$ and $S_{1} \cup \ldots \cup S_{n}=\bar{S}$, where $\bar{S}$ is the minimal factor-closed set containing $S$ (the factor closure of $S$ ). Let $S_{k}=$ $\left\{y_{k, 1}, \ldots, y_{k, p_{k}}\right\}(1 \leq k \leq n)$ and $m=p_{1}+\ldots+p_{n}$ where $y_{k, 1}<\ldots<$ 
$y_{k, p_{k}}=x_{k}$. For $1 \leq j \leq m$, let

$$
y_{j}= \begin{cases}y_{1, j} & \text { if } 1 \leq j \leq p_{1}, \\ y_{k, t} & \text { if } j=p_{1}+\ldots+p_{k-1}+t\left(k \geq 2,1 \leq t \leq p_{k}\right) .\end{cases}
$$

Thus $\bar{S}=\left\{y_{1}, \ldots, y_{m}\right\}$. Let the $n \times m$ matrices $A=\left(a_{i j}\right)$ and $B=\left(b_{i j}\right)$ be defined as follows:

$$
a_{i j}= \begin{cases}g\left(x_{i} / y_{j}\right) \sqrt{f\left(y_{j}\right)} & \text { if } y_{j} \mid x_{i} \\ 0 & \text { otherwise }\end{cases}
$$

and

$$
b_{i j}= \begin{cases}h\left(x_{i} / y_{j}\right) \sqrt{f\left(y_{j}\right)} & \text { if } y_{j} \mid x_{i}, \\ 0 & \text { otherwise. }\end{cases}
$$

It follows immediately from Lemma 1 that

$$
\left[\Psi\left(x_{i}, x_{j}\right)\right]=A B^{\mathrm{T}} .
$$

Let $\left\{\alpha_{1}, \ldots, \alpha_{n}\right\}$ and $\left\{\beta_{1}, \ldots, \beta_{n}\right\}$ denote the systems of row vectors of $A$ and $B$ respectively. Let $\left\{\gamma_{1}, \ldots, \gamma_{n}\right\}$ and $\left\{\delta_{1}, \ldots, \delta_{n}\right\}$ denote the orthogonalization systems obtained from $\left\{\alpha_{1}, \ldots, \alpha_{n}\right\}$ and $\left\{\beta_{1}, \ldots, \beta_{n}\right\}$ respectively by using the Gram-Schmidt orthogonalization process (see [7]), then we have (where $\langle\beta, \beta\rangle$ denotes the inner product)

$$
\left\{\begin{aligned}
\gamma_{1} & =\alpha_{1}, \\
\gamma_{2} & =\alpha_{2}-\frac{\left\langle\alpha_{2}, \gamma_{1}\right\rangle}{\left\langle\gamma_{1}, \gamma_{1}\right\rangle} \gamma_{1} \\
& \vdots \\
\gamma_{n} & =\alpha_{n}-\frac{\left\langle\alpha_{n}, \gamma_{1}\right\rangle}{\left\langle\gamma_{1}, \gamma_{1}\right\rangle} \gamma_{1}-\ldots-\frac{\left\langle\alpha_{n}, \gamma_{n-1}\right\rangle}{\left\langle\gamma_{n-1}, \gamma_{n-1}\right\rangle} \gamma_{n-1}
\end{aligned}\right.
$$

and

$$
\left\{\begin{aligned}
\delta_{1} & =\beta_{1}, \\
\delta_{2} & =\beta_{2}-\frac{\left\langle\beta_{2}, \delta_{1}\right\rangle}{\left\langle\delta_{1}, \delta_{1}\right\rangle} \delta_{1}, \\
& \vdots \\
\delta_{n} & =\beta_{n}-\frac{\left\langle\beta_{n}, \delta_{1}\right\rangle}{\left\langle\delta_{1}, \delta_{1}\right\rangle} \delta_{1}-\ldots-\frac{\left\langle\beta_{n}, \delta_{n-1}\right\rangle}{\left\langle\delta_{n-1}, \delta_{n-1}\right\rangle} \delta_{n-1} .
\end{aligned}\right.
$$

Therefore

$$
\left(\begin{array}{c}
\alpha_{1} \\
\alpha_{2} \\
\vdots \\
\alpha_{n}
\end{array}\right)=\left(\begin{array}{cccc}
1 & 0 & \ldots & 0 \\
\frac{\left\langle\alpha_{2}, \gamma_{1}\right\rangle}{\left\langle\gamma_{1}, \gamma_{1}\right\rangle} & 1 & \ldots & 0 \\
\ldots & \ldots & \ldots & \ldots \\
\frac{\left\langle\alpha_{n}, \gamma_{1}\right\rangle}{\left\langle\gamma_{1}, \gamma_{1}\right\rangle} & \frac{\left\langle\alpha_{n}, \gamma_{2}\right\rangle}{\left\langle\gamma_{2}, \gamma_{2}\right\rangle} & \ldots & 1
\end{array}\right)\left(\begin{array}{c}
\gamma_{1} \\
\gamma_{2} \\
\vdots \\
\gamma_{n}
\end{array}\right)
$$


and

$$
\left(\begin{array}{c}
\beta_{1} \\
\beta_{2} \\
\vdots \\
\beta_{n}
\end{array}\right)=\left(\begin{array}{cccc}
1 & 0 & \ldots & 0 \\
\frac{\left\langle\beta_{2}, \delta_{1}\right\rangle}{\left\langle\delta_{1}, \delta_{1}\right\rangle} & 1 & \ldots & 0 \\
\ldots & \ldots & \ldots & \ldots \\
\frac{\left\langle\beta_{n}, \delta_{1}\right\rangle}{\left\langle\delta_{1}, \delta_{1}\right\rangle} & \frac{\left\langle\beta_{n}, \delta_{2}\right\rangle}{\left\langle\delta_{2}, \delta_{2}\right\rangle} & \ldots & 1
\end{array}\right)\left(\begin{array}{c}
\delta_{1} \\
\delta_{2} \\
\vdots \\
\delta_{n}
\end{array}\right)
$$

Let $M$ and $N$ be the left matrices on the right-hand sides of equations (3) and (4) respectively. Then

$$
\left(\begin{array}{c}
\alpha_{1} \\
\alpha_{2} \\
\vdots \\
\alpha_{n}
\end{array}\right)\left(\begin{array}{llll}
\beta_{1}^{\mathrm{T}} & \beta_{2}^{\mathrm{T}} & \ldots & \beta_{n}^{\mathrm{T}}
\end{array}\right)=M\left(\begin{array}{c}
\gamma_{1} \\
\gamma_{2} \\
\vdots \\
\gamma_{n}
\end{array}\right)\left(\begin{array}{llll}
\delta_{1}^{\mathrm{T}} & \delta_{2}^{\mathrm{T}} & \ldots & \delta_{n}^{\mathrm{T}}
\end{array}\right) N^{\mathrm{T}} .
$$

It follows from (2) and (5) that

$$
\left[\Psi\left(x_{i}, x_{j}\right)\right]=M\left(\begin{array}{c}
\gamma_{1} \\
\gamma_{2} \\
\vdots \\
\gamma_{n}
\end{array}\right)\left(\begin{array}{llll}
\delta_{1}^{\mathrm{T}} & \delta_{2}^{\mathrm{T}} & \ldots & \delta_{n}^{\mathrm{T}}
\end{array}\right) N^{\mathrm{T}} .
$$

Since $x_{1}<\ldots<x_{n}$, it is easy to see that

$$
\left(\alpha_{1}\right)^{(i)}= \begin{cases}g\left(x_{1} / y_{1, i}\right) \sqrt{f\left(y_{1, i}\right)} & \text { if } 1 \leq i \leq p_{1} \\ 0 & \text { if } i>p_{1}\end{cases}
$$

and

$$
\left(\beta_{1}\right)^{(i)}= \begin{cases}h\left(x_{1} / y_{1, i}\right) \sqrt{f\left(y_{1, i}\right)} & \text { if } 1 \leq i \leq p_{1}, \\ 0 & \text { if } i>p_{1}\end{cases}
$$

and for $k \geq 2, i>p_{1}+\ldots+p_{k-1}$, we have

$$
\left(\alpha_{k}\right)^{(i)}= \begin{cases}g\left(x_{k} / y_{k, t}\right) \sqrt{f\left(y_{k, t}\right)} & \text { if } i=p_{1}+\ldots+p_{k-1}+t\left(1 \leq t \leq p_{k}\right), \\ 0 & \text { if } i>p_{1}+\ldots+p_{k}\end{cases}
$$

and

$$
\left(\beta_{k}\right)^{(i)}= \begin{cases}h\left(x_{k} / y_{k, t}\right) \sqrt{f\left(y_{k, t}\right)} & \text { if } i=p_{1}+\ldots+p_{k-1}+t\left(1 \leq t \leq p_{k}\right), \\ 0 & \text { if } i>p_{1}+\ldots+p_{k} .\end{cases}
$$

Thus for $i=p_{1}+\ldots+p_{k-1}+t\left(k \geq 2,1 \leq t \leq p_{k}\right)$, we have

$$
\left(\gamma_{k}\right)^{(i)}=g\left(\frac{x_{k}}{y_{k, t}}\right) \sqrt{f\left(y_{k, t}\right)} \text { and }\left(\delta_{k}\right)^{(i)}=h\left(\frac{x_{k}}{y_{k, t}}\right) \sqrt{f\left(y_{k, t}\right)} .
$$

To complete the proof of Lemma 2, we need the following: 
Lemma 3. With the above notations, let $S=\left\{x_{1}, \ldots, x_{n}\right\}$ be gcd-closed. If $g \in L_{S}$, then

$$
\gamma_{1}=\left(g\left(\frac{x_{1}}{y_{1,1}}\right) \sqrt{f\left(y_{1,1}\right)}, \ldots, g\left(\frac{x_{1}}{y_{1, p_{1}}}\right) \sqrt{f\left(y_{1, p_{1}}\right)}, 0, \ldots, 0\right),
$$

and for $k \geq 2$, we have

$$
\gamma_{k}=(\underbrace{0, \ldots, 0}_{p_{1}+\ldots+p_{k-1}}, g\left(\frac{x_{k}}{y_{k, 1}}\right) \sqrt{f\left(y_{k, 1}\right)}, \ldots, g\left(\frac{x_{k}}{y_{k, p_{k}}}\right) \sqrt{f\left(y_{k, p_{k}}\right)}, 0, \ldots, 0) .
$$

Similarly, if $h \in L_{S}$, then

$$
\delta_{1}=\left(h\left(\frac{x_{1}}{y_{1,1}}\right) \sqrt{f\left(y_{1,1}\right)}, \ldots, h\left(\frac{x_{1}}{y_{1, p_{1}}}\right) \sqrt{f\left(y_{1, p_{1}}\right)}, 0, \ldots, 0\right),
$$

and for $k \geq 2$, we have

$$
\delta_{k}=(\underbrace{0, \ldots, 0}_{p_{1}+\ldots+p_{k-1}}, h\left(\frac{x_{k}}{y_{k, 1}}\right) \sqrt{f\left(y_{k, 1}\right)}, \ldots, h\left(\frac{x_{k}}{y_{k, p_{k}}}\right) \sqrt{f\left(y_{k, p_{k}}\right)}, 0, \ldots, 0) .
$$

Proof. Since the case $h \in L_{S}$ is similar to the case $g \in L_{S}$, we only consider the latter. We argue by induction on $k$. Clearly Lemma 3 is true for $\gamma_{1}$ (since $\left.\gamma_{1}=\alpha_{1}\right)$. Since $S$ is gcd-closed, $\left(x_{2}, x_{1}\right)=x_{1}$. Note that $g \in L_{S}$ implies

$$
g\left(\frac{x_{2}}{y_{1, j}}\right)=g\left(\frac{x_{1}}{y_{1, j}}\right) \quad \text { for } 1 \leq j \leq p_{1} .
$$

Thus

$$
\begin{array}{r}
\alpha_{2}=\left(g\left(\frac{x_{1}}{y_{1,1}}\right) \sqrt{f\left(y_{1,1}\right)}, \ldots, g\left(\frac{x_{1}}{y_{1, p_{1}}}\right) \sqrt{f\left(y_{1, p_{1}}\right)}, g\left(\frac{x_{2}}{y_{2,1}}\right) \sqrt{f\left(y_{2,1}\right)}, \ldots,\right. \\
\left.g\left(\frac{x_{2}}{y_{2, p_{2}}}\right) \sqrt{f\left(y_{2, p_{2}}\right)}, 0, \ldots, 0\right) .
\end{array}
$$

Then $\left\langle\alpha_{2}, \gamma_{1}\right\rangle=\left\langle\gamma_{1}, \gamma_{1}\right\rangle$. Therefore

$$
\begin{aligned}
\gamma_{2} & =\alpha_{2}-\frac{\left\langle\alpha_{2}, \gamma_{1}\right\rangle}{\left\langle\gamma_{1}, \gamma_{1}\right\rangle} \gamma_{1}=\alpha_{2}-\gamma_{1} \\
& =(\underbrace{0, \ldots, 0}_{p_{1}}, g\left(\frac{x_{2}}{y_{2,1}}\right) \sqrt{f\left(y_{2,1}\right)}, \ldots, g\left(\frac{x_{2}}{y_{2, p_{2}}}\right) \sqrt{f\left(y_{2, p_{2}}\right)}, 0, \ldots, 0) .
\end{aligned}
$$

So the assertion is true for $\gamma_{2}$. Suppose that it is true for $\gamma_{l}, 1 \leq l \leq k-1$ $(k \geq 3)$. Now consider $\gamma_{k}$. Since $g \in L_{S}$, we have

$$
\left(\alpha_{k}-\frac{\left\langle\alpha_{k}, \gamma_{1}\right\rangle}{\left\langle\gamma_{1}, \gamma_{1}\right\rangle} \gamma_{1}\right)^{(i)}=0, \quad 1 \leq i \leq p_{1}
$$


We claim that for each $e \in\{2, \ldots, k-1\}$ and each $i$ with $p_{1}+\ldots+p_{e-1}$ $<i \leq p_{1}+\ldots+p_{e}$, we have

$$
\left(\alpha_{k}-\frac{\left\langle\alpha_{k}, \gamma_{e}\right\rangle}{\left\langle\gamma_{e}, \gamma_{e}\right\rangle} \gamma_{e}\right)^{(i)}=0
$$

In fact, if $\left(x_{k}, x_{e}\right)=x_{e}$, then $x_{e} \mid x_{k}$. Note that $g \in L_{S}$ implies $g\left(x_{k} / y_{e, i}\right)=$ $g\left(x_{e} / y_{e, i}\right)$ for $1 \leq i \leq p_{e}$. Thus $\left\langle\alpha_{k}, \gamma_{e}\right\rangle=\left\langle\gamma_{e}, \gamma_{e}\right\rangle$. Hence for each $i$ with $p_{1}+\ldots+p_{e-1}<i \leq p_{1}+\ldots+p_{e}$, we have

$$
\left(\alpha_{k}-\frac{\left\langle\alpha_{k}, \gamma_{e}\right\rangle}{\left\langle\gamma_{e}, \gamma_{e}\right\rangle} \gamma_{e}\right)^{(i)}=\left(\alpha_{k}-\gamma_{e}\right)^{(i)}=0
$$

If $\left(x_{k}, x_{e}\right)=x_{r}$ for some $1 \leq r<e$, then $y_{e, i} \nmid x_{k}$ for all $1 \leq i \leq p_{e}$. Otherwise, there exists $i, 1 \leq i \leq p_{e}$, such that $y_{e, i} \mid x_{k}$. So $y_{e, i} \mid x_{r}$. However, as $r<e$ we have $y_{e, i} \nmid x_{r}$. This is a contradiction. Thus for $p_{1}+\ldots+p_{e-1}<$ $i \leq p_{1}+\ldots+p_{e},\left(\alpha_{k}\right)^{(i)}=0$. So $\left\langle\alpha_{k}, \gamma_{e}\right\rangle=0$. Hence for $p_{1}+\ldots+p_{e-1}<$ $i \leq p_{1}+\ldots+p_{e}$, we have

$$
\left(\alpha_{k}-\frac{\left\langle\alpha_{k}, \gamma_{e}\right\rangle}{\left\langle\gamma_{e}, \gamma_{e}\right\rangle} \gamma_{e}\right)^{(i)}=\left(\alpha_{k}\right)^{(i)}=0 .
$$

This completes the proof of the claim.

Thus it follows from the induction hypothesis and the claim that

$$
\begin{aligned}
\gamma_{k} & =\alpha_{k}-\frac{\left\langle\alpha_{k}, \gamma_{1}\right\rangle}{\left\langle\gamma_{1}, \gamma_{1}\right\rangle} \gamma_{1}-\ldots-\frac{\left\langle\alpha_{k}, \gamma_{k-1}\right\rangle}{\left\langle\gamma_{k-1}, \gamma_{k-1}\right\rangle} \gamma_{k-1} \\
& =(\underbrace{0, \ldots, 0}_{p_{1}+\ldots+p_{k-1}}, g\left(\frac{x_{k}}{y_{k, 1}}\right) \sqrt{f\left(y_{k, 1}\right)}, \ldots, g\left(\frac{x_{k}}{y_{k, p_{k}}}\right) \sqrt{f\left(y_{k, p_{k}}\right)}, 0, \ldots, 0) .
\end{aligned}
$$

The proof of Lemma 3 is complete.

Now we continue to prove Lemma 2. Since $g \in L_{S}$ or $h \in L_{S}$, it follows from Lemma 3 that

$$
\text { (7) } \begin{aligned}
& \left(\begin{array}{c}
\gamma_{1} \\
\gamma_{2} \\
\vdots \\
\gamma_{n}
\end{array}\right)\left(\begin{array}{lllll}
\delta_{1}^{\mathrm{T}} & \delta_{2}^{\mathrm{T}} & \ldots & \delta_{n}^{\mathrm{T}}
\end{array}\right) \\
= & \left(\begin{array}{cccc}
\sum_{j=1}^{p_{1}} f\left(y_{1, j}\right) g\left(\frac{x_{1}}{y_{1, j}}\right) h\left(\frac{x_{1}}{y_{1, j}}\right) & 0 & \ldots & 0 \\
* & \sum_{j=1}^{p_{2}} f\left(y_{2, j}\right) g\left(\frac{x_{2}}{y_{2, j}}\right) h\left(\frac{x_{2}}{y_{2, j}}\right) & \ldots & 0 \\
\vdots & & \vdots & \vdots \\
* & * & \ldots & \sum_{j=1}^{p_{n}} f\left(y_{n, j}\right) g\left(\frac{x_{n}}{y_{n, j}}\right) h\left(\frac{x_{n}}{y_{n, j}}\right)
\end{array}\right)
\end{aligned}
$$




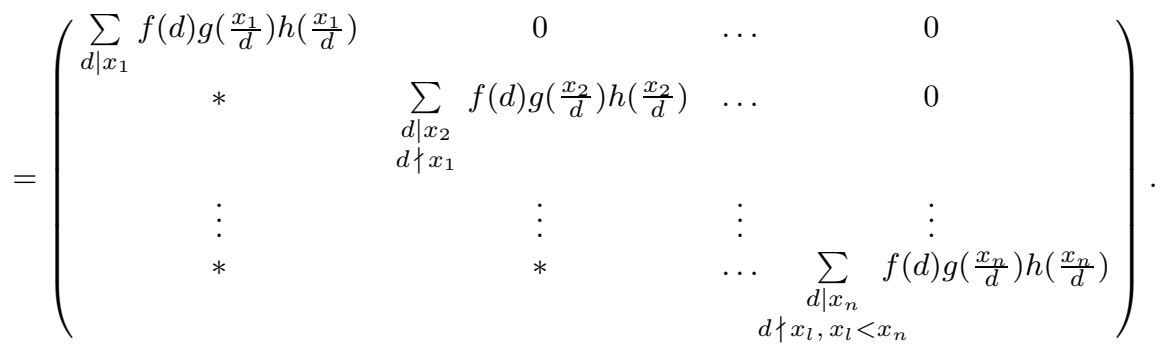

Let

$$
P=\left(\begin{array}{c}
\gamma_{1} \\
\gamma_{2} \\
\vdots \\
\gamma_{n}
\end{array}\right)\left(\begin{array}{llll}
\delta_{1}^{\mathrm{T}} & \delta_{2}^{\mathrm{T}} & \ldots & \delta_{n}^{\mathrm{T}}
\end{array}\right)
$$

By (6) we have $\left[\Psi\left(x_{i}, x_{j}\right)\right]=M P N^{\mathrm{T}}$. Clearly the matrices $M$ and $N$ are lower triangular matrices with diagonal elements 1 . By (7), $P$ is a lower triangular matrix with diagonal elements

$$
\sum_{\substack{d \mid x_{k} \\ d \nmid x_{l}, x_{l}<x_{k}}} f(d) g\left(\frac{x_{k}}{d}\right) h\left(\frac{x_{k}}{d}\right), \quad k=1, \ldots, n .
$$

This completes the proof of Lemma 2.

Now we are ready to give the main result of this paper.

TheOREM 1. Let $S=\left\{x_{1}, \ldots, x_{n}\right\}$ be gcd-closed. If $g \in L_{S}$ or $h \in L_{S}$, then

$$
\operatorname{det}\left[\Psi\left(x_{i}, x_{j}\right)\right]=\prod_{k=1}^{n} \sum_{\substack{d \mid x_{k} \\ d \nmid x_{l}, x_{l}<x_{k}}} f(d) g\left(\frac{x_{k}}{d}\right) h\left(\frac{x_{k}}{d}\right) .
$$

Proof. Since $S=\left\{x_{1}, \ldots, x_{n}\right\}$ is gcd-closed and $g \in L_{S}$ or $h \in L_{S}$, by Lemma 2 there exist $n \times n$ lower triangular matrices $M$ and $N$ with diagonal elements 1 and an $n \times n$ lower triangular matrix $P$ with diagonal elements

$$
\sum_{\substack{d \mid x_{k} \\ d \nmid x_{l}, x_{l}<x_{k}}} f(d) g\left(\frac{x_{k}}{d}\right) h\left(\frac{x_{k}}{d}\right), \quad k=1, \ldots, n,
$$

such that $\left[\Psi\left(x_{i}, x_{j}\right)\right]=M P N^{\mathrm{T}}$. Thus

$$
\operatorname{det}\left[\Psi\left(x_{i}, x_{j}\right)\right]=(\operatorname{det} M)(\operatorname{det} P)\left(\operatorname{det} N^{\mathrm{T}}\right) \text {. }
$$

Note that $\operatorname{det} M=\operatorname{det} N=1$. So $\operatorname{det} N^{\mathrm{T}}=1$. Note also that

$$
\operatorname{det} P=\prod_{k=1}^{n} \sum_{\substack{d \mid x_{k} \\ d \nmid x_{l}, x_{l}<x_{k}}} f(d) g\left(\frac{x_{k}}{d}\right) h\left(\frac{x_{k}}{d}\right) \text {. }
$$

It follows that (8) holds. 
REMARK 1. Let $S=\left\{x_{1}, \ldots, x_{n}\right\}$ be gcd-closed. If $g \in L_{S}$ or $h \in L_{S}$, then (8) gives a formula for $\operatorname{det}\left[\Psi\left(x_{i}, x_{j}\right)\right]$. If $g, h \notin L_{S}$, then we also expect to have a formula for $\operatorname{det}\left[\Psi\left(x_{i}, x_{j}\right)\right]$. This problem remains open.

3. Applications. In this section, we give some interesting applications of our main result.

Theorem 2. Let $S=\left\{x_{1}, \ldots, x_{n}\right\}$ be gcd-closed. If $\beta$ is defined for positive integers $t$ and $r$ by

$$
\beta(t, r)=\sum_{d \mid(t, r)} f(d) g\left(\frac{r}{d}\right),
$$

then

$$
\operatorname{det}\left[\beta\left(x_{i}, x_{j}\right)\right]=\prod_{k=1}^{n} \sum_{\substack{d \mid x_{k} \\ d \nmid x_{l}, x_{l}<x_{k}}} f(d) g\left(\frac{x_{k}}{d}\right) .
$$

Proof. Let $h=\zeta$, where $\zeta$ is defined by $\zeta(d)=1$ for all integers $d$. Clearly $\zeta \in L_{S}$. Then $\Psi(t, r)=\beta(t, r)$. Thus the result follows immediately from Theorem 1.

Remark 2. If $S=\{1, \ldots, n\}$, then Theorem 2 becomes Apostol's result $[2]$.

Corollary 1. If $S=\left\{x_{1}, \ldots, x_{n}\right\}$ is gcd-closed, then

$$
\operatorname{det}\left[C\left(x_{i}, x_{j}\right)\right]=\prod_{k=1}^{n} \sum_{\substack{d \mid x_{k} \\ d \nmid x_{l}, x_{l}<x_{k}}} d \mu\left(\frac{x_{k}}{d}\right) .
$$

Proof. Ramanujan's trigonometric sum $C(t, r)$ is defined by

$$
C(t, r)=\sum_{\substack{k(\bmod r) \\(k, r)=1}} \exp \left(\frac{2 \pi i t}{k}\right)=\sum_{d \mid(t, r)} d \mu\left(\frac{r}{d}\right) .
$$

So if we set $f(d)=d$ for all $d, g=\mu$, then this corollary follows from Theorem 2.

Define the quotient function $\frac{f}{g}$ by

$$
\frac{f}{g}(m)=\frac{f(m)}{g(m)} \quad \text { for positive integers } m \text {. }
$$

Lemma 4 ([1, Theorem 8.8]). Let $f$ be completely multiplicative. Let $g(m)=\mu(m) h(m)$, where $h$ is multiplicative. Assume that $f(p) \neq 0$ and 
$f(p) \neq h(p)$ for all primes $p$. Then

$$
\sum_{d \mid(t, k)} f(d) g\left(\frac{k}{d}\right)=F(k) \frac{g}{F}(N)
$$

where $F=f * g$, and $N=k /(t, k)$.

TheOREM 3. Let $f$ be completely multiplicative. Let $g(m)=\mu(m) h(m)$, where $h$ is multiplicative. Assume that $f(p) \neq 0$ and $f(p) \neq h(p)$ for all primes $p$. If $S=\left\{x_{1}, \ldots, x_{n}\right\}$ is gcd-closed and $(f * g)(d) \neq 0$ for any positive integer d satisfying $d \mid x, x \in S$, then

$$
\operatorname{det}\left[\frac{g}{f * g}\left(\frac{x_{i}}{\left(x_{i}, x_{j}\right)}\right)\right]=\prod_{k=1}^{n} \frac{1}{(f * g)\left(x_{k}\right)} \sum_{\substack{d \mid x_{k} \\ d \nmid x_{l}, x_{l}<x_{k}}} f(d) g\left(\frac{x_{k}}{d}\right) .
$$

Proof. Let $s(k, t)=\sum_{d \mid(t, k)} f(d) g(k / d)$. From Lemma 4, one can deduce that

$$
\begin{aligned}
{\left[s\left(x_{i}, x_{j}\right)\right] } & =\left[(f * g)\left(x_{i}\right) \cdot \frac{g}{f * g}\left(\frac{x_{i}}{\left(x_{i}, x_{j}\right)}\right)\right] \\
& =\operatorname{diag}\left((f * g)\left(x_{1}\right), \ldots,(f * g)\left(x_{n}\right)\right) \cdot\left[\frac{g}{f * g}\left(\frac{x_{i}}{\left(x_{i}, x_{j}\right)}\right)\right] .
\end{aligned}
$$

Thus we have

$$
\operatorname{det}\left[\frac{g}{f * g}\left(\frac{x_{i}}{\left(x_{i}, x_{j}\right)}\right)\right]=\operatorname{det}\left[s\left(x_{i}, x_{j}\right)\right] \prod_{k=1}^{n} \frac{1}{(f * g)\left(x_{k}\right)} .
$$

Therefore the result follows from the above equation and Theorem 2.

Corollary 2. Let $S=\left\{x_{1}, \ldots, x_{n}\right\}$ be factor-closed, and let the arithmetical functions $f$ and $g$ be as in Theorem 3. Then

$$
\operatorname{det}\left[\frac{g}{f * g}\left(\frac{x_{i}}{\left(x_{i}, x_{j}\right)}\right)\right]=[g(1)]^{n} \prod_{k=1}^{n} \frac{f}{f * g}\left(x_{k}\right) .
$$

Corollary 3. Let the arithmetical functions $f$ and $g$ be as in Theorem 3. Then

$$
\operatorname{det}\left[\frac{g}{f * g}\left(\frac{i}{(i, j)}\right)\right]=[g(1)]^{n} \prod_{k=1}^{n} \frac{f}{f * g}(k) .
$$

An arithmetical function $f(t)$ is said to be quadratic if it is the Dirichlet convolution of two completely multiplicative functions $[9,12]$. In what follows we use Theorem 1 and the following result of Vaidyanathaswamy, concerning quadratic functions, to evaluate the determinants of $n \times n$ matrices of the form $\left[f\left(x_{i} x_{j}\right)\right]$, where $f(t)$ is a quadratic function. 
LEMma 5 (Vaidyanathaswamy [12]). If $f=g * h$ where $g$ and $h$ are completely multiplicative functions, then $f$ satisfies the identity

$$
f(t, r)=\sum_{d \mid(t, r)} f\left(\frac{t}{d}\right) f\left(\frac{r}{d}\right) g(d) h(d) \mu(d) .
$$

THEOREM 4. Let $f=g * h$, where $g$ and $h$ are completely multiplicative. If $S=\left\{x_{1}, \ldots, x_{n}\right\}$ is gcd-closed and $f \in L_{S}$, then

$$
\operatorname{det}\left[f\left(x_{i} x_{j}\right)\right]=\prod_{k=1}^{n} \sum_{\substack{d \mid x_{k} \\ d \nmid x_{l}, x_{l}<x_{k}}} g(d) h(d) \mu(d)\left[f\left(\frac{x_{k}}{d}\right)\right]^{2} .
$$

Proof. This result follows from Lemma 5 and Theorem 1.

LEMma 6 ([10]). The arithmetical function $f$ is a semi-multiplicative function if and only if for any positive integers $m$ and $n, f(m) f(n)=$ $f((m, n)) f([m, n])$.

Lemma 7. Let $f$ be an arithmetical function. Then for any positive integer $n$,

$$
\sum_{d \mid n}(f * \mu)(d)=f(n)
$$

Proof. Let the arithmetical function $I$ be defined for any positive integer $m$ as follows: $I(m)=[1 / m]$, where $[x]$ denotes the greatest integer not greater than $x$. Since $\mu * \zeta=I$ (see [1]) and $f=f * I$, one has

$$
\begin{aligned}
f(n) & =(f * I)(n)=(f *(\mu * \zeta))(n)=((f * \mu) * \zeta)(n) \\
& =\sum_{d \mid n}(f * \mu)(d) \zeta\left(\frac{n}{d}\right)=\sum_{d \mid n}(f * \mu)(d),
\end{aligned}
$$

as desired. The proof of Lemma 7 is complete.

THEOREM 5. Let $f$ be a semi-multiplicative function and $f[t, r]$ denote $f$ evaluated at the least common multiple of $t$ and $r$. If $S=\left\{x_{1}, \ldots, x_{n}\right\}$ is gcd-closed, then

$$
\operatorname{det}\left(f\left[x_{i}, x_{j}\right]\right)=\prod_{k=1}^{n}\left[f\left(x_{k}\right)\right]^{2} \sum_{\substack{d \mid x_{k} \\ d \nmid x_{l}, x_{l}<x_{k}}}\left(\frac{1}{f} * \mu\right)(d) .
$$

Proof. Since $f$ is semi-multiplicative, it follows from Lemma 6 that

$$
\left(f\left[x_{i}, x_{j}\right]\right)=D\left(g\left(x_{i}, x_{j}\right)\right) D,
$$

where $g=1 / f$ and $D=\operatorname{diag}\left(f\left(x_{1}\right), \ldots, f\left(x_{n}\right)\right)$. Thus

$$
\operatorname{det}\left(f\left[x_{i}, x_{j}\right]\right)=\operatorname{det}\left[g\left(x_{i}, x_{j}\right)\right] \prod_{k=1}^{n}\left[f\left(x_{k}\right)\right]^{2} .
$$


Let $g=h=\zeta$ and substitute $g * \mu$ for $f$ in Theorem 1. By Lemma 7, one has $\Psi\left(x_{i}, x_{j}\right)=g\left(x_{i}, x_{j}\right)$. Thus it follows from Theorem 1 that

$$
\operatorname{det}\left(g\left[x_{i}, x_{j}\right]\right)=\prod_{k=1}^{n} \sum_{\substack{d \mid x_{k} \\ d \nmid x_{l}, x_{l}<x_{k}}}(g * \mu)(d) .
$$

It then follows from (10) and (11) that (9) holds.

Remark 3. If we set $f(d)=d$ for all integers $d$, then Theorem 5 reduces to Bourque and Ligh's result [4]. Bourque and Ligh [4] conjectured that the LCM matrix $\left(\left[x_{i}, x_{j}\right]\right)$ defined on a gcd-closed set $S=\left\{x_{1}, \ldots, x_{n}\right\}$ is nonsingular. We showed [5] that the Bourque-Ligh conjecture is true for a certain class of gcd-closed sets $S=\left\{x_{1}, \ldots, x_{n}\right\}$. We proved [6] that the Bourque-Ligh conjecture is true if $n \leq 7$, but not true if $n \geq 8$. We believe that this result is true for general positive integer power LCM matrices. We conclude this paper by raising the following conjecture.

Conjecture. Let $m$ be a given positive integer and $n$ any positive integer. Then there is a positive integer $k(m)$, depending only on $m$, such that if $n \leq k(m)$, then the power LCM matrix $\left(\left[x_{i}, x_{j}\right]^{m}\right)$ defined on any gcd-closed set $S=\left\{x_{1}, \ldots, x_{n}\right\}$ is nonsingular. But for $n \geq k(m)+1$, there exists a gcd-closed set $S=\left\{x_{1}, \ldots, x_{n}\right\}$ so that the power LCM matrix $\left(\left[x_{i}, x_{j}\right]^{m}\right)$ defined on $S$ is singular.

From [6], one knows that the above conjecture holds when $m=1$. In fact, $k(1)=7$. In a similar way to [6], one can show that for any integer $m \geq 2$, one has $k(m) \geq 7$.

Acknowledgements. The author wishes to thank the referee for his careful reading of the manuscript and helpful suggestions.

\section{References}

[1] T. Apostol, Introduction to Analytic Number Theory, Springer, New York, 1976.

[2] -, Arithmetical properties of generalized Ramanujan sums, Pacific J. Math. 41 (1972), 281-293.

[3] K. Bourque and S. Ligh, Matrices associated with classes of arithmetical functions, J. Number Theory 45 (1993), 367-376.

[4] -, - On GCD and LCM matrices, Linear Algebra Appl. 174 (1992), 65-74.

[5] S. F. Hong, On LCM matrices on GCD-closed sets, Southeast Asian Bull. Math. 22 (1998), 381-384.

[6] -, On the Bourque-Ligh conjecture of least common multiple matrices, J. Algebra 218 (1999), 216-228.

[7] P. Lancaster and M. Tismenetsky, The Theory of Matrices, 2nd ed., Academic Press, New York, 1985. 
[8] P. J. McCarthy, A generalization of Smith's determinant, Canad. Math. Bull. 29 (1988), 109-113.

[9] K. G. Ramanathan, Multiplicative arithmetic functions, J. Indian Math. Soc. 7 (1943), 111-116.

[10] D. Rearick, Semi-multiplicative functions, Duke Math. J. 33 (1966), 49-53.

[11] H. J. S. Smith, On the value of a certain arithmetical determinant, Proc. London Math. Soc. 7 (1875-76), 208-212.

[12] R. Vaidyanathaswamy, The identical equations of the multiplicative function, Bull. Amer. Math. Soc. 36 (1930), 762-772.

Mathematical College

Sichuan University

Chengdu 610064

P.R. China

E-mail: hongsf@263.net 